\title{
RICHIR LECTEUR DE FINK : MÉTHODE ET ARCHITECTONIQUE
}

STÉPHANE FINETTI

\begin{abstract}
This paper focuses on an important moment in Richir's elaboration of the question of method and architectonic: his confrontation with Fink's phenomenology and especially with Sixth Cartesian Meditation. Moving from Fink's approach to phenomenological reduction and to architectonic in the Sixth Cartesian Meditation, we will see how Richir recasts them in the Sixth Phenomenological Meditation.
\end{abstract}

\section{Introduction}

Nous aborderons la question de la méthode et de l'architectonique dans la phénoménologie de M. Richir par le biais de sa confrontation avec la phénoménologie d'E. Fink et, en particulier, avec la Sixième méditation cartésienne. Cette méditation supplémentaire, rédigée par Fink en 1932 pour parachever son remaniement des Méditations cartésiennes, ébauche en effet l'Idée d'une doctrine transcendantale de la méthode. En tant que telle, elle réactive non seulement le sens général de la deuxième partie principale de la Critique de la raison pure, mais aussi le sens de son troisième chapitre : l'Architectonique de la raison pure. La question de la méthode et de l'architectonique ${ }^{1}$ est en outre précisément celle qui sollicite Richir dans la Sixième méditation:

1 Comme nous le verrons, il ne s'agit pas de deux questions séparées, mais d'une seule et même question.

https://doi.org/10.14712/24646504.2020.19

(C) 2020 The Author. This is an open-access article distributed under the terms of the

Creative Commons Attribution License (http://creativecommons.org/licenses/by/4.0). 
Ce qui me sollicitait alors dans ce texte, c'était évidemment la question de l'architectonique de la phénoménologie, puisque Fink y élabore une théorie transcendantale de la méthode en contrepoint d'une théorie transcendantale des éléments : il reprend explicitement les titres de Kant. ${ }^{2}$

La confrontation de Richir avec la Sixième méditation peut ainsi être considérée comme une partie intégrante de son élaboration de la question de la méthode et de l'architectonique, voire même comme un de ses moments-clés ${ }^{3}$.

On distinguera trois phases dans la rencontre de Richir avec cet ouvrage de Fink. Avant sa publication, Richir ne le connaissait qu'indirectement, à travers la Phénoménologie de la perception de M. Merleau-Ponty. Ce dernier avait pu en effet consulter les $\$ \$ 1-11$ de la Sixième méditation grâce à G. Berger, qui en avait reçu un exemplaire de Husserl en 1934. Merleau-Ponty en avait ainsi fait deux brèves citations dans l'Avant-propos de la Phénoménologie de la perception. La première concernait la "phénoménologie constructive " 5 , abordée par Fink dans le $\$ 7$ de la Sixième méditation, mais présente d'un bout à l'autre de l'ouvrage. La seconde portait sur le thème de la doctrine transcendantale de la méthode : l'ego méditant en tant que "'spectateur impartial' (uninteressierte Zuschauer) »'. L'Avant-propos de la Phénoménologie de la perception se référait aussi implicitement à la question de l'éidétique transcendantale abordée par Fink dans le $\$ 9$ de la Sixième méditation. Ces questions constituent l'horizon à partir duquel Richir entreprit la lecture de la Sixième méditation dès sa publication par H. Ebeling, J. Holl et G. van Kerckhoven dans la série Husserliana-Dokumente. En 1988, s'ouvrait ainsi une deuxième phase de la rencontre de Richir avec la Sixième méditation, caractérisée par deux contributions majeures : en premier lieu, la conférence La question d'une doctrine transcendantale de la méthode en phénoménologie ${ }^{7}$, donnée aux Archives Eugen Fink de l'Université de Fribourg à l'occasion du colloque international Questions fondamentales de la méthode et de la science phénoménologique (1989), où Richir fit la connaissance de G. van Kerckhoven ; en deuxième lieu, l'ouvrage Méditations

2 Richir Marc, L'écart et le rien. Conversations avec Sacha Carlson, Grenoble, Millon, 2015, p. 197.

3 Un autre moment-clé (que nous nous limiterons à évoquer) est constitué par la redéfinition richirienne de l'architectonique comme tectonique de l'archaïque ( $c f$. Richir M., « La refonte de la phénoménologie ", in Annales de phénoménologie, 7, 2008, p. 207).

4 Cf. Van Kerckhoven Guy, Mundanisierung und Individuation, Würzburg, Konigshausen \& Neumann, 2003, p. 79.

5 Merleau-Ponty Maurice, Phénoménologie de la perception, Paris, Gallimard, 1945, p. I.

$6 \quad$ Ibid, p. XV.

7 Richir M., «La question d'une doctrine transcendantale de la méthode en phénoménologie », in Epokhé, 1, 1990, pp. 91-125. 
phénoménologiques (1992) et, notamment, la Sixième méditation phénoménologique ${ }^{8}$, intitulée Phénoménologie et architectonique. Dans les deux cas, Richir mettait en œuvre une lecture phénoménologique et non-doctrinale de la Sixième méditation : une lecture qui ne cherchait pas à l'objectiver comme doctrine historique, mais à mettre en relief ses ressources implicites pour procéder à une refonte de la méthode et de l'architectonique phénoménologiques. Une troisième phase de la rencontre de Richir avec la Sixième méditation est enfin constituée par le travail éditorial qui le conduisit à publier en 1994 - en tant que directeur de la collection Krisis de l'éditeur J. Millon - la traduction française de la Sixième méditation (effectuée par N. Depraz), ainsi qu'en 1998 la traduction française du remaniement finkien des Méditations cartésiennes (effectuée par F. Dastur et A. Montavont).

L' élaboration de la question de la méthode et de l'architectonique que Richir entreprend dans la Sixième méditation phénoménologique renvoie aussi à un deuxième volet de sa confrontation avec la phénoménologie d'E. Fink : son analyse du cours Monde et finitude, tenu par Fink à l'Université de Fribourg dans les semestres d'été 1949 et 1966. Le $\$ 3$ a de la Sixième méditation phénoménologique s’y réfère en effet de façon implicite 9 . L'article Monde et phénomènes (1992) ${ }^{10}$ en constitue au contraire une analyse explicite et détaillée. Richir y reprend notamment la distinction heideggerienne, puis finkienne entre Terre et Monde pour la repenser comme distinction architectonique. Ce deuxième volet de la confrontation de Richir avec la phénoménologie d'E. Fink allait de pair avec une recherche des prémisses de Monde et finitude dans les notes de recherche du jeune Fink. Richir fut à notre connaissance le premier à en entreprendre la traduction dans le milieu francophone : il traduisit dans les numéros 15/16 de Les cahiers de philosophie le recueil de notes « Sur le concept phénoménologique de Monde (1930 et 1934) » ainsi que "Esquisses pour l'écrit : Monde et concept du monde, une recherche théorétique du problème (1935) ${ }^{11}$. Ce travail de recherche et de traduction aboutit en 1994 à l'organisation du colloque Eugen Fink (avec N. Depraz) ainsi qu'aux contributions

8 Cf. Richir M., Méditations phénoménologiques, Grenoble, Millon, 1992, pp. 329-380, en particulier les pp. 338-362.

9 Cf. Richir M., Méditations phénoménologiques, op. cit., p. 377.

10 Cf. Richir M., "Monde et phénomènes », in Les Cahiers de Philosophie, 15/16, 1992, pp. 111-137.

$11 C f$. Richir M., «E. Fink - Sur le concept phénoménologique de Monde (1930 et 1934) et «Esquisses pour l'écrit : Monde et concept du monde, une recherche théorétique du problème (1935) ", in Les Cahiers de Philosophie, 15/16, 1992/93, pp. 71-88. 
Temps, espace et monde chez le jeune Fink (1994) ${ }^{12}$ et Schwingung et phénoménalisation (1997) ${ }^{13}$.

Notre contribution s'articulera ainsi en deux parties, consacrées à la question de la méthode et de l'architectonique dans la confrontation de Richir avec la Sixième méditation cartésienne et avec Monde et finitude ${ }^{14}$.

\section{La confrontation avec la Sixième méditation cartésienne}

Comme l'indique son sous-titre - L'idée d'une doctrine transcendantale de la méthode - la Sixième méditation a la tâche de soumettre à l'analyse phénoménologique les méthodes qui opèrent de manière anonyme dans les cinq premières Méditations cartésiennes et, en général, dans la phénoménologie husserlienne. Il s'agit avant tout et surtout de la méthode de la réduction phénoménologique : "le premier problème de la doctrine transcendantale de la méthode [...] non seulement en tant que problème nécessairement introductif, mais en tant que problème fondamental $\aleph^{15}$. La doctrine transcendantale de la méthode consiste ainsi dans l'analyse des différentes formes de réduction phénoménologique mises en œuvre par Husserl et de leur progression méthodique. En distinguant les différents niveaux réductifs à l'œuvre dans la phénoménologie husserlienne, la doctrine transcendantale de la méthode met aussi en relief les problèmes constitutifs qu'ils permettent de poser ainsi que leur progression méthodique. Dans la mesure où cette dernière confère aux problèmes constitutifs une unité systématique, la doctrine transcendantale de la méthode met enfin en lumière " la systématique des questions phénoménologiques ${ }^{16}$. Comme l’explique Fink dans le $\$ 2$ de la Sixième méditation, la question de la méthode réductive « contient [...] toute la

12 Richir M., « Temps, espace et monde chez le jeune Fink », in Depraz Natalie et Richir M. (dir.), Engen Fink - Actes du colloque de Cerisy-La-Salle - 23-30 juillet 1994, Amsterdam, Rodopi, 1997, pp. $27-42$.

13 Richir M., "Schwingung et phénoménalisation ", in Internationale Zeitschrift für Philosophie, 1, 1998, pp. 52-63.

14 On pourrait mentionner également un troisième volet de la confrontation de Richir avec la phénoménologie de Fink : $c f$. Richir M., "Le trait social fondamental du travail humain / E. Fink: Introduction, Traduction, Notes brèves en guise de commentaire ", in Revue Internationale de Psychopathologie du Travail, 2, 1999, pp. 63-74. Nous ne pourrons cependant l'aborder dans ce contexte.

15 Fink Eugen, VI Cartesianische Meditation, Die Idee einer transzendentalen Methodenlehre, Ebeling Hans, Holl Jann et van Kerckhoven G. (dir.), Dordrecht, Kluwer, 1988 (Husserliana-Dokumente II/1, cité dorénavant Hua-Dok II/1), p. 32 ; tr. fr. de Depraz N., Sixième méditation cartésienne. L'idée d'une doctrine transcendantale de la méthode, Grenoble, Millon, 1994, p. 82.

16

Hua-Dok II/1, p. 8 ; tr. fr. p. 60. 
systématique de la philosophie phénoménologique in nuce ${ }^{17}$. En ce sens, elle s'avère être aussi la question de « la systématique et de l'architectonique interne du savoir transcendantal ${ }^{18}$.

Fink reprend l'expression " architectonique » de la Critique de la raison pure, où elle indique justement "l'art des systèmes ${ }^{19}$. Pour Kant, il s'agit de l'art de reconduire "l'unité de diverses connaissances sous une Idée " ${ }^{20}:$ «le concept rationnel de la forme d'un tout, en tant que c'est en lui que sont déterminées a priori la sphère des éléments divers et la position respective des parties ${ }^{21}$. Chez Fink, l'architectonique permet de manière analogue de situer un problème constitutif au sein du système des problèmes phénoménologiques ou, encore, de situer une discipline phénoménologique (par exemple, la phénoménologie constructive) au sein du système de la philosophie phénoménologique. Ce dernier est pour Fink un "système des 'horizons de travail ouverts' $»^{22}$ ou, mieux, un horizon systématique. $S$ 'il considère le système de la philosophie phénoménologique comme un « espace problématique clos ${ }^{23}$, c'est en effet pour préciser aussitôt que :

[...] la clôture du système phénoménologique ne signifie nullement la possibilité d'un parcours rapide, d'une maîtrise du système par quelques formules fondamentales. La phénoménologie n'est pas un dire pensant, architectoniquement clos, esthétiquement satisfaisant, mais une philosophie de travail. Elle a devant elle un travail analytique infini, un horizon infiniment ouvert de recherches concrètes. ${ }^{24}$

Dans la mesure où elle concerne un horizon, la clôture du système phénoménologique est paradoxalement aussi son ouverture : Fink peut ainsi affirmer, d'une part, que « en son principe, un tel système ne peut qu'être que clos » ${ }^{25}$ et, d'autre

17 Hua-Dok II/1, p. 10 ; tr. fr. p. 62.

18 Hua-Dok II/1, p. 110 ; tr. fr. p. 156.

19 Kant Immanuel, Critique de la raison pure, Paris, PUF, 2014, p. 558.

20 Idem.

21 Idem.

22 Fink E., VI Cartesianische Meditation, Teil 2 - Ergänzungsband, Dordrecht, Kluwer, 1988 (Husserliana-Dokumente II/2, cité dorénavant Hua-Dok II/2), p. 3; tr. fr. de F. Dastur et A. Montavont, Autres rédactions des Méditations cartésiennes, Grenoble, Millon, 1998, p. 15.

23 Fink E., Studien zur Phänomenologie 1930-1939, Den Haag, Nijhoff, 1966 (Phaenomenologica 21, cité dorénavant Phaen 21), p. 177 ; tr. fr. de Franck Didier, De la phénoménologie, Paris, Minuit, 1974, p. 197.

24 Phaen 21, p. 177 ; tr. fr. pp. 196-197.

25 Phaen 21, p. 177 ; tr. fr. p. 196. 
part, qu'il s'agit d'un « système ouvert ${ }^{26}$. En tant qu'horizon systématique, le système de la philosophie phénoménologique est enfin caractérisé par une «mobilité » ${ }^{27}$ (Beweglichkeit) essentielle. D’une part, il oriente les analyses phénoménologiques concrètes et leur fournit leur site architectonique. D'autre part, il croît dans les analyses phénoménologiques concrètes : elles le débordent et l'obligent ainsi à se modifier dans une dynamique qui demeure toujours ouverte. ${ }^{28}$ Le système de la philosophie phénoménologique est un système en mouvement.

Dans la mesure où Fink réactive le sens de la doctrine transcendantale de la méthode de Kant et, en particulier, de l'architectonique de la raison pure, Richir reconnaît en lui un précurseur ${ }^{29}$. Il entreprend ainsi dans La doctrine transcendantale de la méthode en phénoménologie et dans les $\$ \$ 1 \mathrm{~b}$-c de la Sixième méditation phénoménologique une lecture phénoménologique et non-doctrinale de l'architectonique de la Sixième méditation cartésienne : l'architectonique de la phénoménologie de Husserl selon Fink. À cette fin, Richir se concentre sur son articulation tripartite, que Fink ébauche dans la Disposition du «système de philosophie phénoménologique »d'Edmund Husserl (1930) et expose dans le $\$ 2$ de la Sixième méditation (1932). En reprenant les titres des parties principales de la Critique de la raison pure, Fink articule le système de la philosophie phénoménologique en esthétique transcendantale, en analytique transcendantale (que Fink appelle aussi phénoménologie descriptive ou régressive) et en dialectique transcendantale (que Fink appelle aussi phénoménologie constructive ou progressive). La lecture phénoménologique et non-doctrinale que Richir en entreprend est un moment-clé de sa refonte de la phénoménologie husserlienne : elle refond la méthode et l'architectonique de la phénoménologie husserlienne thématisées par Fink dans la Sixième méditation. Dans les termes de Richir,

Tous ces commentaires à propos de la tripartition proposée par Fink de la phénoménologie husserlienne nous permettent de mieux saisir le sens de la refonte que nous en proposons $[\ldots]^{30}$

26 Fink E., Gesamtausgabe, Band 3, Phänomenologische Werkstatt, Teilband 2: Die Bernauer Manuskripte, Cartesianische Meditationen und System der phänomenologischen Philosophie, Bruzina Ronald (dir.), Freiburg, Alber, 2008, p. 333.

27 Idem.

$28 C f$. à ce sujet, G. Chernavin, "L'architectonique flexible de la phénoménologie ", in Annales de phénoménologie 14, 2015, pp. 97-120.

29 Cf. Richir M., "La question d'une doctrine transcendantale de la méthode en phénoménologie », art. cit., p. 93 : «l'étude de la VIe Méditation m’a fait découvrir avec émerveillement un ancêtre bien réel, quoique jusqu'il y a peu inconnu de moi ».

30 Richir M., Méditations phénoménologiques, op. cit., p. 357. 
C'est ce mouvement de refonte que nous chercherons à mettre en évidence dans l'interprétation richirienne de l'esthétique, de l'analytique et de la dialectique transcendantales.

L'esthétique transcendantale est d'après le $\$ 2$ de la Sixième méditation «l'analyse du 'phénomène de monde' $»^{31}$ ou, mieux, «l'analyse des cogitata qua cogitata et de leurs structures universelles $»^{32}$. En tant que telle, l'esthétique transcendantale met en ouvre la réduction éidétique : la variation imaginaire des cogitata en vue de l'intuition éidétique de leur identité de sens. Elle met en lumière (en tant qu'éidétique matérielle) les articulations régionales des étants et (en tant qu'éidétique formelle) leurs articulations catégoriales. Comme Richir le fait remarquer, l'esthétique transcendantale relève en ce sens de la pré-donnée du monde, c'est-àdire de l'attitude naturelle :

C'est très lucidement que, dans le projet de 1930/31 d'exposé systématique, Fink considère l'éidétique comme faisant partie intégrante de la pré-donnée (Vorgegebenheit) du monde. ${ }^{33}$

En tant qu'ontologie régionale et en tant qu'ontologie formelle, l'esthétique transcendantale présuppose en effet l'attitude naturelle comme capture (Befangenheit) dans l'idée d'être mondain ainsi que comme capture dans l'éidétique mondaine. En outre, elle présuppose l'attitude naturelle comme capture dans la langue naturelle, dont " tous les concepts sont des concepts d'être " ${ }^{34}$ et qui est indispensable à l'institution des eide. Dans les termes de Richir, l'esthétique transcendantale présuppose l'institution symbolique de la langue ${ }^{35}$.

Les identités de sens dégagées dans l'esthétique transcendantale sont les fils conducteurs de l'analytique transcendantale, qui en analyse la constitution. L'analytique transcendantale prend tout d'abord la forme de l'analyse statique : l'analyse des différentes formes de corrélation noético-noématique rendues manifestes par la réduction phénoménologique à l'immanence (que Fink appelle aussi «noématisation »). L'analyse statique conduit selon Richir l'institution symbolique de la langue à s'interroger, à travers l'ipse qui est censé la sous-tendre, sur le sens de ses déterminations ${ }^{36}$. L'analytique transcendantale prend ensuite la forme de l'analyse

Hua-Dok II/1, p. 11 ; tr. fr. p. 63.

Idem.

33 Richir M., «La question d'une doctrine transcendantale de la méthode en phénoménologie », art. cit., p. 99.

4 Hua-Dok II/1, p. 94; tr. fr. p. 140.

5 Cf. Richir M., Méditations phénoménologiques, op. cit., p. 351.

36 Ibid., p. 352. 
génétique : l'explicitation des implications intentionnelles des corrélations noético-noématiques. Il s'agit par exemple de l'explicitation de l'excès du sens sur le sens, implicite en toute intentionnalité d'acte :

Il y a dans l'excès du sens sur le sens, en jeu dans toute intentionnalité, du sens qui ne relève pas strictement de l'identité, et qui met en œuvre un champ de sens pluriels, d'autres sens, eux aussi possibles dans la constitution, et qui font la cohésion du monde. ${ }^{37}$

Ce sens en deçà de toute identité de sens n'est pas un sens déjà fait, mais un sens en train de se faire. Il se forme dans les intentionnalités d'horizon du passé et du futur, c'est-à-dire dans la temporalisation, ainsi que dans la spatialisation du Leib. Cette formation de sens (Sinnbildung) est la condition de possibilité de l'institution (Stiftung) de toute identité de sens et de l'intentionnalité d'acte qui la vise.

À ce niveau d'analyse, le sens se faisant et sa formation demeurent cependant encore déformés de façon cohérente par l'institution des identités de sens et des aperceptions dont ils sont censés rendre compte :

L'analytique génétique s'ouvre à l'immensité proprement dite de l'apeiron phénoménologique, mais dans la mesure où, chez Husserl, elle n'est encore conçue qu'en vue de l'explicitation téléologique des sens d'identités, elle y est encore déformée de façon cohérente par ce qui sera, désormais, une articulation fort complexe de téléologies, polarisées par des aperceptions de langue $[\ldots] .^{38}$

En premier lieu, l'analyse génétique (au sens de la phénoménologie descriptive ou régressive) demeure orientée par le fil conducteur que lui a fourni préalablement l'esthétique transcendantale : les identités de sens obtenues par la réduction éidétique. La formation du sens dans la temporalisation et la spatialisation de la conscience demeure ainsi conçue comme constitution téléologiquement orientée vers l'institution d'identités de sens. Ou encore, la formation du sens est conçue comme potentialité d'aperceptions de langue, au lieu d'être comprise comme relevant d'un niveau constitutif transpossible par rapport aux aperceptions de langue ${ }^{39}$.

Ibid., p. 353.

Idem.

39 Richir reprend le concept de transpossibilité de la phénoménologie d'H. Maldiney et, en particulier, de Penser l'homme et la folie (qu'il fait paraître en 1991 dans la collection Krisis de l'éditeur J. Millon). Comme son nom l'indique, la trans-possibilité nomme chez Maldiney « ce qui s'ouvre au-delà ou en deçà de tout possible et qui au regard de la pensée positiviste est impossible " (H. Maldiney, Penser l'homme et la folie, Millon, Grenoble, 2007, p. 228). Dans le cadre de sa refonte richirienne, 
Ce n'est que la dialectique transcendantale ou phénoménologie constructive qui offre la possibilité de suspendre cette surdétermination du champ phénoménologique par l'institution symbolique de la langue : une possibilité qui selon Richir est entrevue et, en même temps, manquée par Fink. De même que la dialectique transcendantale de Kant concerne des Idées qui par principe ne peuvent être exhibées en aucune expérience sensible, la phénoménologie constructive concerne en effet ce qui par principe échappe à toute donation intuitive (immanente et, a fortiori, transcendante) : elle «n' est rien d'autre que le concept intrinsèque de toutes les positions de problèmes qui vont au-delà de la donnée réductive de la vie transcendantale ${ }^{40}$. En ce sens, la phénoménologie constructive ouvre au champ phénoménologique en tant qu'originairement non-donné et permet de repenser son rapport à l'institution symbolique comme " rapport de la donnée phénoménologique (éidétique ou téléologique) à une non-donnée originaire » ${ }^{41}$. Fink l'exemplifie par le commencement et la fin de la vie transcendantale (égologique et intersubjective). Le commencement de la vie égologique (le passé de naissance) ou, encore, le commencement de la vie intersubjective (d'avant la naissance) ne peuvent par principe jamais être donnés dans le re-souvenir : ils sont un passé immémorial, qui n’a jamais été présent. De même, la fin de la vie égologique (le futur de la mort) ou, encore, la fin de la vie intersubjective (après la mort) ne peuvent par principe jamais être données dans le pro-souvenir : elles sont un futur à jamais immature, qui ne sera jamais présent. Le commencement et la fin de la vie (égologique et intersubjective) se constituent pour Fink dans les déprésentations (Entgegenwärtigungen) des horizons du passé et du futur, à leur tour inconvertibles en re-souvenirs ou en pro-souvenirs immanents ${ }^{42}$. La phénoménologie constructive s'ouvre ainsi à la proto-temporalisation des phénomènes-de-monde, à partir de laquelle doit être repensée la temporalisation du sens se faisant. Dans les termes de Richir,

ce concept permet de penser la différence architectonique entre le registre de la Sinnbildung et celui de la Sinnstiftung. La formation du sens dans la temporalisation de la conscience et dans la spatialisation du Leib est la condition de possibilité de l'institution des aperceptions : par exemple, des aperceptions de langue. Le rapport entre Sinnbildung et Sinnstiftung n'est cependant pas celui de la puissance à l'acte : la Sinnbildung n'est pas une simple potentialité d'aperceptions et ces dernières ne sont pas le télos de la Sinnbildung. Si c'était le cas, rien d'imprévu ne pourrait surgir. La Sinnbildung relève au contraire d'un autre registre architectonique que la Sinnstiftung : elle excède aussi bien l'actualité des aperceptions (par exemple des aperceptions de langue) que leurs potentialités et leur demeure en ce sens transpossible. Sur ce concept, que Richir repense successivement comme virtuel, $c f$. I. Fazakas \& E. Bellato, «Le virtuel et le transcendantal», in Metodo. International Studies in Phenomenology and Philosophy, t. II, 2, 2014, pp. 203-225, en particulier pp. 212-218.

40 Hua-Dok II/1, p. 66 ; tr. fr. p. 114.

41 Richir M., «La question d'une doctrine transcendantale de la méthode en phénoménologie », art. cit., p. 106.

42 Cf. Richir M., « Temps, espace et monde chez le jeune Fink », art. cit., p. 29. 
elle s'ouvre à une proto-temporalisation hors langage, à laquelle est référée la temporalisation du sens se faisant en tant que temporalisation de langage.

Pour éviter toute surdétermination du champ phénoménologique (de langage et hors langage) par l'institution symbolique de la langue, la seule possibilité est de mettre en œuvre une réduction phénoménologique radicale : une réduction phénoménologique plus radicale que la réduction à l'immanence (dans sa forme égologique ou intersubjective). Elle prend avant tout pour Fink la forme de la déshumanisation (Entmenschung) : la mise en suspens de l'ipse symboliquement institué et de ses habitus. Elle se présente en outre comme réduction de l'idée d'être mondain et comme réduction de l'éidétique mondaine, c'est-à-dire comme mise en suspens de l'opposition mondaine entre fait et essence. Fink considère en effet comme une erreur fondamentale le fait de « comprendre la nature de la réduction à partir de l'éidétique [...] au lieu, à l'inverse, de poser la question de la nature de l'eidos transcendantal comme problème issu de l'accomplissement de la réduction ${ }^{43}$. La réduction de l'idée d'être et de l'éidétique s'avèrent impliquer enfin une réduction de la langue, qui rend possible l'institution des essences mondaines et des faits mondains : "La réduction de l'idée d'être impliquée par la réduction phénoménologique a ainsi pour conséquence [...] une réduction de la langue ${ }^{44}$.

Dans les trois cas (la déshumanisation, la réduction de l'idée d'être mondain et de l'éidétique mondaine, la réduction de la langue), Fink « touche» selon Richir " comme par tangence ${ }^{45}$ au sublime kantien et, dans la mesure où il ouvre au champ phénoménologique, au sublime phénoménologique. La déshumanisation, motivée par l'effroi, évoque « la première partie [...] du moment kantien du sublime $»^{46}$ : celle où l'ipse, en mourant comme ipse symboliquement institué, fait l'épreuve de sa propre énigme. La réduction de l'idée d'être mondain (qui met en suspens tout étant transcendant ou immanent) et la réduction de l'éidétique mondaine (qui met en suspens toute essence transcendante ou immanente) ouvre au champ phénoménologique comme Rien transcendantal : « Le connaître phénoménologisant n'est-il en définitive référé à 'rien' ? » ${ }^{47}$. Le spectateur phénoménologique n'y a paradoxalement plus rien à voir. De même, la réduction de la langue «place bien d'une certaine manière le 'spectateur' qui vient d'être établi devant le

\footnotetext{
Phaen 21, 138 ; tr. fr. p. 159. Cf. aussi Richir M., Méditations phénoménologiques, op. cit., p. 338.

Hua-Dok II/1, p. 103 ; tr. fr. p. 149.

Cf. Richir M., Méditations phénoménologiques, op. cit., p. 356 et « La question d’une doctrine transcendantale de la méthode en phénoménologie», art. cit., pp. 116-117.

Idem.

Cf. Hua-Dok II/1, p. 80; tr. fr. p. 126.
} 
rien ${ }^{48}$ : le Rien transcendantal, face auquel il demeure "stupéfait » et « muet » $(\text { spachlos })^{49}$. Dans les termes de Richir,

[...] l'épochè radicale comme ébranlement transcendantal est tout à la fois épochè de l'ipse lui-même, où celui-ci ressort comme une énigme, et épochè de l'être qui débouche sur le Rien (I, 80), où, s'il y a encore un « spectateur transcendantal », celui-ci n'a plus rien à voir, est aveugle, et n'a plus rien à dire, est sans langage (sprachlos) ou muet (I, $105)^{50}$.

Cette épochè phénoménologique radicale, sous l'exposant de laquelle Richir place ce qu'il a " avancé pour refondre et agrandir la phénoménologie ${ }^{51}$, ouvre au champ phénoménologique des phénomènes comme rien que phénomènes : des phénomènes qui ne sont l'apparition ou la donation de rien et qui, de plus, n'apparaissent pas et ne se donnent pas ${ }^{52}$.

Pourtant, Fink finit selon Richir par manquer le sublime phénoménologique qui se dessine en creux dans la déshumanisation, dans la réduction de l'idée d'être mondain, dans la réduction de l'éidétique et dans la réduction de la langue ${ }^{53}$. En premier lieu, il manque l'ouverture à l'absolument grand qui se produit chez Kant dans le sublime mathématique et l'ouverture à l'absolument puissant qui se produit chez Kant dans le sublime dynamique. En traversant par la déshumanisation sa mort en tant qu'ipse symboliquement institué, l'ipse devrait faire non seulement l'épreuve de sa propre énigme, mais aussi l'épreuve de «l'énigme de ce qui l'institue dans son identité non-conceptuelle et non-éidétique " ${ }^{54}:$ "l'énigme de ce qui, [...], par delà les bords du monde et ses horizons, est ce que nous nommons l'instituant symbolique ${ }^{55}$. En deuxième lieu, Fink perd par là-même le champ phénoménologique des phénomènes comme rien que phénomènes, qui s'éclipsent selon Richir dans leur simulacre ontologique : le Rien transcendantal prend la forme de l'être (ou du pré-être) transcendantal. Comme l'explique Richir,

\footnotetext{
Cf. Hua-Dok II/1, p. 103; tr. fr. p. 149.

Cf. Hua-Dok II/1, p. 105; tr. fr. p. 151.

Richir M., «La question d'une doctrine transcendantale de la méthode en phénoménologie », art. cit., p. 111.

Ibid., p. 110.

Cf. Ibid., pp. 118-119.

Cf. Ibid., p. 117.

Idem.

Idem.
} 
[...] manquant le moment d'un sublime phénoménologique, la VIe Méditation nous propose l'architectonique d'une institution symbolique, celle de la phénoménologie husserlienne, sans instituant symbolique, et [...] c'est cela, sans nul doute, qui se convertit, par le simulacre ontologique ou la précédence ontologique de l'ipse véritable, en au moins l'ombre ou le fantôme d'une onto-théologie où l'être " originaire " est censé porter tout le poids de l'être « dérivé » $[\ldots]^{56}$.

Et le fantôme de l'onto-théologie va de pair selon Richir avec celui de l'auto-transparence de l'être originaire ${ }^{57}$.

Comme le précise la Sixième méditation phénoménologique, ce qui pour Richir fait l'intérêt de la Sixième méditation cartésienne n'est constitué cependant ni par les hauts-fonds ni par les récifs où Fink risque de faire naufrage, mais par le voyage qu'il entreprend $^{58}$. Richir cherche à le prolonger en réactivant le sens du sublime, de la dialectique et de l'architectonique de Kant. Il entreprend ainsi une triple refonte : 1 . une refonte de la réduction phénoménologique comme "épreuve phénoménologique du sublime ${ }^{59} ; 2$. une refonte de la dialectique transcendantale en tant que clignotement inhérent aux phénomènes comme rien que phénomènes ; 3. une refonte de l'architectonique de la philosophie phénoménologique. Pour ce qui concerne le premier point, Richir qualifie d'abord l'épreuve phénoménologique du sublime comme le « suspens $»^{60}$ de toute temporalisation et de toute spatialisation de langage, c'est-à-dire de tout schématisme de langage. Ou, encore, l'épreuve phénoménologique du sublime consiste dans une "paralysie ou une stupeur de tout phénomène de langage ${ }^{61}$ : elle est « l'épreuve qu’à trop [...] posséder » les sens, « on les perd à raison même de la transparence qu'ils semblent avoir acquise " ${ }^{62}$. L'ipséité du sens s'évanouit dès lors dans l'apeiron phénoménologique des phénomènes-de-monde, faisant surgir ainsi l'énigme de son origine à partir de la proto-temporalisation et de la proto-spatialisation, c'est-à-dire à partir du

56 Ibid., pp. 118-119.

57 La Sixième méditation cartésienne ou, mieux, la métaphysique phénoménologique qui en est le couronnement a souvent été considérée comme une onto-théologie. Cette approche (que nous avons partagée) nous semble réductive à la lumière des recherches actuelles : la métaphysique phénoménologique du jeune Fink peut être interprétée de manière non onto-théologique. On remarquera que Richir laisse ici ouverte cette possibilité dans la mesure où il n'identifie pas la Sixième méditation avec son ombre ou son fantôme onto-théologique. Cf. Richir M., Méditations phénoménologiques, op. cit., p. 358.

59 Richir M., «La question d'une doctrine transcendantale de la méthode en phénoménologie », art. cit., p. 122.

60 Richir M., Méditations phénoménologiques, op. cit., p. 362.

61 Idem.

62 Ibid., p. 371. 
schématisme hors langage. L'épreuve phénoménologique du sublime fait surgir en outre l'énigme de l'origine de l'ipséité du soi. Pour le soi symboliquement institué et, plus radicalement, pour le soi incarné qui assiste au schématisme de langage, elle consiste en effet dans une traversée de la mort :

[...] le sublime phénoménologique est ce "moment " phénoménologique où, [...], l'ipse fait l'épreuve phénoménologique de sa propre mort, l'épreuve de l'inhabitation et de l'inhabitabilité du phénomène-de-monde par le soi, de ce que le phénomène-de-monde ne m'a jamais attendu en sa phénoménalité et ne m’attendra jamais, mais me fait au contraire glisser vers l'absence et la non-donation [...]. Par là, [...] l'ipse se découvre essentiellement comme énigme $[\ldots]^{63}$.

L'énigme à laquelle ouvre le sublime phénoménologique est cependant plus fondamentalement celle de "l'instituant symbolique, à distance du monde, au-delà de ses bords et plus profond que ses entrailles " ${ }^{64}$. L'épreuve phénoménologique du sublime est l'ouverture à un Autre radical qui nous habite : non pas un Dieu présent ou un Dieu cadavre, mais " une absence indéfiniment en fuite dans la transcendance d'absence du monde $»^{65}$.

De même qu'il refond la réduction phénoménologique en réactivant le sens du sublime kantien, Richir refond la dialectique transcendantale introduite par Fink dans le $\$ 2$ de la Sixième méditation:

Où serait donc, pour nous, le «lieu » d'une dialectique transcendantale ? Tout d'abord, rappelons que, pour nous, les questions de la naissance et de la mort se posent au lieu du sublime phénoménologique $[\ldots]^{66}$.

Si on la repense à partir de l'épreuve phénoménologique du sublime, la dialectique transcendantale ne peut cependant plus se réduire à une doctrine de la totalité de la vie transcendantale (égologique ou intersubjective). Elle doit être repensée au contraire comme inhérente au champ phénoménologique en tant que tel. Plus précisément, l'illusion transcendantale qui nous fait prendre les totalités de la vie (égologique et intersubjective) pour des données phénoménologiques alors qu'elles sont radicalement non-données, doit être considérée comme inhérente

63 Cf. Richir M., "La question d'une doctrine transcendantale de la méthode en phénoménologie », art. cit., pp. 119-120.

64 Ibid., p. 120.

65 Cf. Richir M., Méditations phénoménologiques, op. cit., p. 372.

66 Idem. 
à tout phénomène comme rien que phénomène : ce dernier ne se phénoménalise qu'en clignotant incessamment entre son surgissement comme non-donné originaire et son évanouissement dans l'illusion transcendantale de sa donation intuitive.

Richir met au jour cette dialectique transcendantale phénoménologique de deux manières dans la Sixième méditation phénoménologique. En premier lieu, il se confronte avec les appendices de Husserl à la Sixième méditation cartésienne. Les appendices XIII et XIV, en particulier, lui permettent de dégager le clignotement des phénomènes comme rien que phénomènes, ainsi que celui du soi phénoménologisant. Le champ de la phénoménalité ne se phénoménalise qu'en clignotant incessamment entre son surgissement « comme quelque chose qui ne s'est pas déjà mondanisé » et son évanouissement " venant de ce que, si le surgissement s'accomplit, ce qui surgit prend immédiatement les traits aperceptifs du 'mondain' ${ }^{67}$. Les phénomènes de langage, par exemple, se phénoménalisent en clignotant entre leur surgissement comme sens se faisant et leur évanouissement dans les identités de sens instituées de la langue. Pour cette raison, les phénomènes comme rien que phénomènes ne peuvent être aperçus par le soi phénoménologisant, mais seulement entre-aperçus dans leur clignotement : ils ne peuvent être qu' entrevus dans le double mouvement qui les empêche de s'engloutir dans du disparu et de s'épanouir dans de l'apparu. Leur entre-aperception est précisément pour Richir «le schème-organe de la phénoménalisation " ${ }^{68}$. Pour la même raison, le soi phénoménologisant (aveugle et muet) ne peut s'installer de façon durable dans l'attitude transcendantale : il clignote incessamment entre l'attitude transcendantale où surgit le champ phénoménologique et l'attitude naturelle où il s'évanouit dans son illusion transcendantale.

\section{La confrontation avec Monde et finitude}

Le deuxième ouvrage avec lequel Richir se confronte pour mettre au jour la dialectique transcendantale des phénomènes comme rien que phénomènes est le cours Monde et finitude, tenu par Fink à l'Université de Fribourg dans les semestres d'été 1949 et 1966. Ce cours se situe dans un autre contexte que la Sixième méditation cartésienne (1932). En effet, il est l'aboutissement des recherches théorétiques et historiques sur le concept de monde entreprises par Fink au milieu

67 Ibid., p. 361.

68 Ibid., p. 362. 
des années 30, en vue d'un projet d'habilitation alternatif à l'Idée d'une doctrine transcendantale de la méthode. En outre, Monde et finitude inaugure une nouvelle perspective par rapport à la Sixième méditation cartésienne ainsi qu'aux notes de recherche des années 30 : celle d'une phénoménologie cosmologique. Avec le $₫ 3 \mathrm{a}$ de la Sixième méditation phénoménologique et, surtout, avec l'article Monde et phénomènes ${ }^{69}$, Richir est à notre connaissance le premier à avoir entrepris une analyse détaillée de ce cours dans le monde francophone. De même, il est le premier à avoir entrepris une traduction des notes de recherche dont il est l'aboutissement : il traduisit dans les numéros 15/16 de Les cahiers de philosophie le recueil de notes «Sur le concept phénoménologique de Monde (1930 et 1934)» (dont l'original est publié aujourd'hui dans Phänomenologische Werkstatt I/II) et « Esquisses pour l'écrit : Monde et concept du monde, une recherche théorétique du problème (1935)» (dont l'original demeure aujourd'hui encore inédit). ${ }^{70}$

Ce qui sollicite Richir dans Monde et finitude n'est pas premièrement la question de la méthode et de l'architectonique : il s'agit avant tout et surtout de la question du monde, qu'il considère " plus originelle que la question de l'être $»^{71}$. Bien qu'elle ne soit pas au premier plan, la question de la méthode et de l'architectonique émerge néanmoins régulièrement dans la lecture richirienne de Monde et finitude. Dans le $\$ 23$ de la troisième section du cours, intitulée Discussion non-métaphysique du concept de monde, par exemple, l'attention de Richir est attirée par le passage suivant :

[...] nous ne nous comportons pas envers le monde de la manière la plus véridique si nous avons une vue d'ensemble la plus grande possible de l'étant; mais si le Tout vibre (schwingt) à travers notre être-là, [...] si nous nous laissons saisir par le «ciel étoilé au-dessus de moi » et le « regard sur la haute mer ${ }^{72}$

Comme Fink l'ajoute plus loin, le monde se phénoménalise véritablement lorsqu'il vibre (schwingt) en nous dans « le sentiment du sublime (das Gefühl des

69 Cf. Richir M., " Monde et finitude », art. cit., pp. 113-137.

70 Cf. Richir M., «E. Fink - Sur le concept phénoménologique de Monde (1930 et 1934) et «Esquisses pour l'écrit : Monde et concept du monde, une recherche théorétique du problème (1935) ", in Les Cahiers de Philosophie, 15/16, 1992/93, pp. 71-88.

71 Cf. Richir M., " Monde et phénomènes », art. cit., p. 113.

72 Fink E., Welt und Endlichkeit, Würzburg, Könighausen \& Neumann, 1990, p. 203: « [...] zur Welt verhalten wir uns nicht dann am wahrhaftesten, wenn wir einen möglichst großen Überblick über das Seiende haben; sondern wenn das Ganze durch unser Dasein schwingt, [...] wenn wir uns auslassen in der Ergriffenheit, die „der bestirnte Himmel über mir“ und der „Blick auf ferne Meere“ uns ins Herz schickt ». 
Erhabenen) ${ }^{73}$ suscité par le ciel étoilé au-dessus de nous ou par le regard sur la haute mer. Par ce renvoi au moment kantien du sublime, Fink entrevoit selon Richir " une sorte d'épochè phénoménologique-cosmologique » ${ }^{74}$ : la mise en suspens " de toute chose ou de tout événement, de tout étant, en laquelle parait, à travers l'épreuve de la mort, quelque chose comme le monde même $»^{75}$.

Fink ne pratique pourtant pas selon Richir cette épochè phénoménologique-cosmologique jusqu'au bout. Bien qu'il repense la différence ontologique heideggerienne comme différence cosmologique et l'éclaircie (Lichtung) de l'être comme éclaircie du monde, il demeure en effet encore tributaire de « la conception heideggerienne de la phénoménalité comme phénoménalité de quelque chose qui est $»^{76}$. Une réactivation conséquente du sens du sublime kantien l'aurait conduit au contraire selon Richir à remédier à cette " relative cécité architectonique ${ }^{77}$. En premier lieu, elle l'aurait amené à analyser les antinomies cosmologiques et, en général, la dialectique transcendantale autrement que dans les $\$ \$ 12-14$ de Monde et finitude. Fink y aborde en effet la dialectique transcendantale à travers l'axe exclusif de la question du monde, comme si les questions de l'ipse et de Dieu avaient été réglées. Une réactivation conséquente du sens du sublime kantien aurait exigé au contraire que Fink écrive non seulement Welt und Endlichkeit, mais " en même temps, pour ainsi dire, Selbstheit und Endlichkeit et Gott und Endlichkeit ${ }^{78}$. L'étude de la reprise de la problématique des antinomies cosmologiques de la première Critique dans la problématique du sublime de la troisième Critique aurait en outre conduit Fink à reconnaître qu' "il y a là un véritable traitement phénoménologique de l'illusion transcendantale de la raison pure théorique $»^{79}$, qui rend l'illusion transcendantale indissociable des phénomènes comme rien que phénomènes. Enfin, cette réactivation du sens de l'illusion transcendantale aurait exigé une refonte de l'éclaircie (Lichtung) de l'être heideggerienne comme éclaircie du monde indissociable de son illusion transcendantale. ${ }^{80}$

\footnotetext{
Fink E., Welt und Endlichkeit, op. cit., p. 203.

Richir M., « Monde et phénomènes », art. cit., p. 113.

Ibid., p. 116.

Ibid., p. 114.

Idem.

Idem.

Ibid., p. 116.

80 Cf. Idem : « D'un point de vue kantien [...] la régression heideggerienne vers l'Ungrund d'où s'enlève le Grund de la fondation - régression admirablement reprise par Fink, depuis le monde comme existential jusqu'à l'ek-stase originaire rendant elle-même possible l'existential - a tous les caractères, moyennant un autre concept, distinct de la causalité, de l'enchaînement des conditions, de la régression du conditionné à l'inconditionné, sans que, jamais, chez Heidegger, cela ne donne lieu à une "Dialectique transcendantale", où serait, sinon réfléchi, du moins soupçonné, que la
} 
C'est précisément ce que Richir cherche à mettre en œuvre dans Monde et phénomènes et dans le $\$ 3$ a de la Sixième méditation phénoménologique, en prolongeant « le sens architectonique kantien, très aigu chez Fink dans la Sixième méditation $[. .$.$] jusque dans Heidegger { }^{81}$. Dans les termes du dernier paragraphe de Monde et phénomènes, intitulé Monde, illusion transcendantale et phénomènes,

La question qui se pose est la suivante : la Lichtung [...] est-elle foncièrement distincte de l'illusion transcendantale dès lors que l'illusion transcendantale n'est plus à concevoir comme illusion transcendantale d'étant ?2 $^{82}$

La possibilité de concevoir l'illusion transcendantale autrement que comme illusion de l'étant découle ici de la remise en question richirienne de l'idée de cèlement (Verbergung) exposée par Heidegger dans L'origine de l'œuvre d'art. Heidegger distingue en effet dans cet ouvrage deux modes de cèlement : le refus (Versagen) et le déplacement (Verstellen). En tant que refus, le cèlement est « le commencement de l'éclaircie (Lichtung) de l'éclairci (Gelichtete) » ${ }^{83}$. Il est " le refus de l'étant dont nous ne pouvons encore dire rien d'autre que : il est ${ }^{84}$. En tant que déplacement (Verstellen), le cèlement a lieu au contraire au milieu de l'éclairci et consiste dans les « glissements, voilements, obscurcissements, masquages, dénégations de l'étant par de l'étant ${ }^{85}$. On ne saurait discerner selon Heidegger ces deux modes de cèlement avec certitude : le cèlement se cèle et se déplace lui-même. Il ne concerne ainsi pour lui que l'éclairci (Gelichtete) et non pas l'éclaircie (Lichtung) elle-même. Richir remet en question l'indistinction de ces deux formes de cèlement et, surtout, la limitation du déplacement à la seule sphère ontique : " est-on sûr que les 'phénomènes' du Verstellen n'aient lieu qu' avec des 'apparaissants' ? ». ${ }^{86}$

À travers l'épreuve phénoménologique du sublime, les phénomènes du Verstellen s'avèrent relever plutôt du monde au sens archaïque, sauvage et barbare. Ou encore, dans les termes de L'origine de l'œuvre d'art, que Fink reprend et radicalise dans Monde et finitude, ils relèvent de la Terre : le monde au sens cosmique. Alors qu'au monde au sens existential appartiennent les étants, au monde au sens cosmique appartiennent les Wesen sauvages ou concrétudes phénoménologiques.

Lichtung elle-même - et l'Ereignis - puissent à leur tour être une illusion transcendantale de la pensée ».

81 Ibid., p. 117.

82 Ibid., pp. 132-133. Cf. aussi ibid., p. 116.

83 Ibid., p. 128.

84 Idem.

85 Ibid., p. 129.

86 Ibid., p. 130. 
Avant d'intéresser l'étant, le cèlement au sens du déplacement (Verstellen) intéresse donc les Wesen sauvages ou concrétudes phénoménologiques. Richir parvient ainsi à un nouveau concept d'éclaircie (Lichtung) :

Si le champ phénoménologique, dans sa dimension immémoriale et primordiale, en prend les caractères de " monde " archaïque, sauvage, barbare, de phénonènes-demonde agités de multitudes, de myriades de déplacements de leurs concrétudes phénoménologiques, il ne peut plus être milieu de Lichtung au même sens que celui de Heidegger. S'il y a Lichtung, c'est, encore une fois, celle, océanique, cosmique, panique, ou dionysiaque, du sublime $[\ldots]{ }^{87}$

S'il y a Lichtung il ne peut plus s'agir de l'éclaircie de l'être, mais d'une éclaircie du monde indissociable de son illusion transcendantale. En ce sens, pour Richir, " les phénomènes et la phénoménalité ne sont pas primairement 'comptables' de la question de l'être ${ }^{88}$.

Richir repense ainsi la distinction heideggerienne, puis finkienne entre Terre et Monde comme distinction architectonique entre, d'une part, le champ des phénomènes-de-monde et, d'autre part, le monde symboliquement institué. Comme il l'explique lui-même dans Monde et phénomènes,

Nous reconnaissons [...] dans la Terre, et dans l' ' intuition » finkienne qu' elle est le monde au sens cosmique, tous les caractères de ce que nous avons repéré comme l'inconscient phénoménologique. Et dans le "monde " au sens heideggerien tous les caractères du monde en tant qu'il est symboliquement institué - et symboliquement codé - dans une culture. Heidegger, mais sans doute encore Fink, avec cette « intuition ", ont été au plus près de "découvrir » les phénomènes-de-monde, au sens où nous l'entendons $[\ldots] .{ }^{89}$

Dans la mesure où leur distinction est repensée comme distinction architectonique entre le champ des phénomènes-de-monde et le monde symboliquement institué, Terre et Monde sont cependant caractérisés par une " hétérogénéité bien plus abyssale que ne le soupçonnait Heidegger ${ }^{90}$. Il y a désormais entre la Terre

\footnotetext{
Richir M., Méditations phénoménologiques, op. cit., p. 377.

Idem.

Richir M., Monde et phénomènes, art. cit., p. 126.

Ibid., p. 131.
} 
(relevant du registre phénoménologique) et le Monde (relevant du registre symbolique) un "abîme infranchissable " ${ }^{91}$.

\section{Conclusion}

La confrontation avec la Sixième méditation cartésienne (dont Richir loue la clairvoyance architectonique) et avec Monde et finitude (dont Richir déplore la relative cécité architectonique) aboutit ainsi dans le $\$ 3$ b de la Sixième méditation phénoménologique à une refonte de l'architectonique de la phénoménologie husserlienne. Nous en exposerons pour conclure les traits principaux, en les confrontant avec ceux de l'architectonique de la phénoménologie husserlienne selon Fink.

En premier lieu, l'architectonique de la phénoménologie ne relève plus pour Richir de l'ontologie. Par exemple, elle ne prévoit plus comme la Disposition du "système de philosophie phénoménologique » d'Edmund Husserl ébauchée par Fink en 1930 une distinction des niveaux de la phénoménologie pure (régressive, progressive, etc.) et des niveaux d'être (transcendantal, mondain, etc.). Elle concerne au contraire le champ archaïque des phénomènes comme rien que phénomènes et leur phénoménalisation ${ }^{92}$ : d'une part, le schématisme phénoménologique (de langage et hors langage) et, d'autre part, le proto-ontologique (les Wesen sauvages de langage et hors langage). Ce n'est que par déformation cohérente ou, comme Richir le dira successivement, par transposition architectonique que le proto-ontologique bascule pour lui dans l'ontologique.

En deuxième lieu, l'architectonique ne semble plus pouvoir être définie chez Richir comme une systématique. Comme il l'explique dans le $₫ 3 \mathrm{~b}$ de la Sixième méditation phénoménologique, "l'architectonique [...] n'est pas un 'système' de l'esprit humain (Fichte, Hegel) $»^{93}$. Ou encore,

Il serait illusoire de croire que l'architectonique que nous déployons [...] doive se condenser en la saturation doctrinale d'un " système $» .94$

91 Richir M., Idem. La distinction entre Terre et Monde est également centrale dans la phénoménologie a-subjective de J. Patočka, avec laquelle Richir se confronte (entre autres) dans la Troisième méditation phénoménologique. Sur la distinction entre Terre et Monde (ou Terre et Ciel) chez Patočka, cf. O. Stanciu, « Nature et monde naturel dans la pensée de J. Patočka », in Alter 26, 2018, pp. 47-64.

92 Cf. Ibid., p. 318.

93 Ibid., p. 377.

94 Ibid., p. 379. 
L'architectonique des problèmes phénoménologiques ne peut se condenser en un système parce qu'ils demeurent fondamentalement des "problèmes à résoudre ${ }^{95}$ et même des problèmes qui « en un sens, ne seront jamais résolus ${ }^{96}$. D'une part, en effet, ils passent inaperçus. D’autre part, leur résolution demanderait " une durée de vie excédant largement la durée de la vie humaine $»^{97}$.

Si l'architectonique ne peut se condenser en la saturation doctrinale d'un système clos, ne peut-on cependant la rapprocher du système des horizons de travail ouverts ébauché par Fink dans la Disposition du «système de philosophie phénoménologique " d'Edmund Husserl et dans la Sixième méditation cartésienne? Richir opère lui-même ce rapprochement au terme de la Première méditation phénoménologique:

[...] comme Husserl lui-même l'a toujours conçu, la phénoménologie est radicalement hétérogène au système ou à l'esprit de système - et s'il y a un «système » en elle, c'est seulement dans le sens où Fink lui-même l'avait remarquablement compris dans la $V I^{e}$ Méditation cartésienne, c'est-à-dire au sens kantien de l'architectonique, qui n’implique aucune décision métaphysique - du moins en droit, dans la mesure où elle doit être, et rester une architectonique de questions et de problèmes en réalité infinis. ${ }^{98}$

D’après ce passage, il faut entendre en phénoménologie le terme système au sens kantien de l'architectonique, réactivé par Fink dans la Sixième méditation, c'est-à-dire comme architectonique de questions et de problèmes infinis.

Si le système est à repenser en phénoménologie à partir de l'architectonique, Richir ne soutient cependant pas l'inverse (tout au moins, pas dans le même sens du terme système). Bien que l'architectonique finkienne soit sans nul doute au plus près de l'architectonique richirienne, cette dernière en est en effet une refonte et remet en question précisément son caractère systématique (fût-il ouvert). L'épreuve phénoménologique du sublime fait pour ainsi dire éclater la dimension systématique (fût-elle ouverte) de la phénoménologie : elle ouvre au champ phénoménologique comme apeiron phénoménologique et à une transcendance en fuite infinie, qui excèdent tout système possible. Richir déclare ainsi dans le $₫ 3 \mathrm{~b}$ de la Sixième méditation phénoménologique:

\footnotetext{
Idem.

Idem.

Idem.

Cf. Ibid., p. 23.
} 
[...] si l'architectonique est un "système ", c'est un système d'orientations, par surcroît variables selon la question ou le problème envisagés, mais d'orientations selon des axes symboliques. ${ }^{99}$

Si l'architectonique est un système, elle ne l'est plus au sens du système des horizons de travail ouverts de Fink. Elle l'est au contraire en tant que système d'orientations : elle est le système d'axes symboliques qui, depuis l'institution de la langue philosophique, permet de s'orienter dans le champ phénoménologique (donc dans les problèmes phénoménologiques et dans la phénoménologie ellemême). Il ne s'agit cependant en aucun cas d'un système fixe de coordonnées : il varie au contraire « selon la question ou le problème envisagés » ${ }^{100}$.

De cette manière, Richir opère par rapport à l'architectonique de Fink un troisième déplacement. Alors que chez Fink l'architectonique est surtout la systématique du savoir et des problèmes phénoménologiques, elle devient chez Richir avant tout une architectonique du champ phénoménologique (de langage, hors langage) et de sa distinction de l'institution symbolique de la langue. Dans cette architectonique refondue, la langue, le langage et le hors langage ne sont plus des « couches » (Schichten) intentionnelles, mais des « registres » ${ }^{101}$ : Richir distingue le registre phénoménologique (de langage et hors langage) du registre symbolique de la langue. L'architectonique richirienne se distingue en outre de toute "stratigraphie » ${ }^{102}$ des couches intentionnelles. Alors que dans cette dernière les couches les plus profondes sont les plus anciennes et les couches les plus superficielles les plus récentes, dans l'architectonique richirienne les couches sont sujettes à déformation (superposition, retournement, etc.) : « les couches les plus anciennes », par exemple, "peuvent passer au-dessus de couches bien plus récentes » ${ }^{103}$. Ou encore, les registres architectoniques sont sujets à déformation ou transposition : l'institution symbolique des aperceptions de langue va de pair avec la transposition architectonique du schématisme phénoménologique de langage en potentialités d'aperceptions de langue. Cette refonte de l'architectonique se situe dans le prolongement de Phénoménologie et institutions symbolique (1988), où Richir présente l'analyse du champ phénoménologique comme une " géologie transcendantale »104 de l'institution symbolique de la langue. De même que dans l'orogenèse les

\footnotetext{
$99 \quad$ Ibid., p. 378.

100 Idem.

${ }^{101}$ Cf. Richir M., L'écart et le rien, op. cit., p. 193.

102 Cf. Idem.

103 Cf. Idem.

104 Richir M., Phénoménologie et institution symbolique, Grenoble, Millon, 1988, p. 197, 198.
} 
mouvements des plaques tectoniques sont à l'origine des chaînes de montagnes, le schématisme phénoménologique des phénomènes-de-monde (qui correspondent pour Richir à la Terre au sens heideggerien, puis finkien du terme) et le schématisme phénoménologique des phénomènes-de-langage sont en effet à l'origine de l'institution symbolique de la langue. La refonte de l'architectonique mise en œuvre dans la Sixième méditation phénoménologique et l'idée de géologie transcendantale exposée dans Phénoménologie et institution symbolique constituent enfin les prémisses de la redéfinition de l'architectonique comme tectonique que Richir entreprend à partir de L'expérience du penser ${ }^{105}$. Dans La refonte de la phénoménologie (2008), par exemple, Richir la redéfinit comme « tectonique, au sens géologique du terme, de l'archaïque ${ }^{106}$ :

L'architectonique comme méthode est donc une mise en ordre des problèmes et questions selon nos concepts qui, pour l'essentiel, sont des concepts philosophiques [...]. À cela doit correspondre, en principe, l'architectonique comme tectonique de la « chose " (Sache) même, à savoir comme mouvements, chevauchements, ruptures, failles, charriages, etc. de l'archaïque $[\ldots] .107$

En ce sens, l'architectonique n'est plus simplement la "mise en ordre » des problèmes et des questions phénoménologiques, mais aussi et surtout une tectonique (au sens géologique du terme) du champ archaïque des phénomènes comme rien que phénomènes et du schématisme phénoménologique.

Stéphane Finetti est docteur en philosophie de l'Université de Toulouse II et de l'Université de Pise. Il a publié en 2013 Riflessione e astrazione chez Mimesis et en 2014 La phénoménologie de la phénoménologie d'E. Fink et son problème directeur chez Millon. Ses recherches actuelles portent sur l'itinéraire de pensée de Fink et sur la phénoménologie de la phantasia de Husserl, Fink et Richir.

105 Cf. Richir M., L'expérience du penser, Grenoble, Millon, 1996, pp. 57 : « Dans ce passage à travers le 'moment' du sublime, il y a remise en jeu, en profondeur, de l'archi-tectonique des rapports de la langue au langage - en tant que travail tectonique, qui 'cisaille' la langue en éclats intercalés de langage, de l'arché, pourtant toujours déjà perdue, de l'institution symbolique elle-même ». $C f$. aussi ibid., p. 64.

106 Richir M., «La refonte de la phénoménologie », art. cit., p. 207.

107 Ibid., pp. 207-208. 